\title{
Panafrikanismus, transnationaler Schwarzer Feminismus und die Grenzen des kulturalistischen Blicks in afrikanischen Geschlechterdiskursen ${ }^{1}$
}

\section{Mit der "Konjunktur" leben ${ }^{2}$}

Feminismus und Panafrikanismus sind politische Positionen des 20. Jahrhunderts, die sich verschiedentlich überschneiden, in ihrem Kampf gegen vergeschlechtlichte und rassifizierte Herrschaftssysteme aber unterschiedliche Pfade einschlagen. ${ }^{3}$ Aus der Geschichte des Panafrikanismus sind Frauen weitgehend herausgeschrieben worden. Deshalb muss darauf hingewiesen werden, dass der Panafrikanismus von Anfang an auch durch aktive Frauen geprägt wurde. Viele dieser Frauen vertraten in ihrer Arbeit und ihrem Aktivismus ausdrücklich Frauenrechtspositionen. Panafrikanistinnen, die Feministinnen waren (oder Feministinnen, die Panafrikanistinnen waren) beherrschten die Kunst, im Rahmen des großen Ziels der Befreiung afrikanischer Völker international mit verschiedenen komplexen Positionen zu »Rasse«, Geschlecht, Klasse, Nationalität und Kultur zu navigieren. Mit ihren politischen Positionen lassen sie sich in die gegen-

1 Der Text erschien auf Englisch zuerst in: Feminist Africa 19/2014: Pan-Africanism and Feminism, 78-93.

2 Anm. d. Ü.: Carole Boyce Davies gebraucht in diesem Aufsatz das Vokabular der Cultural Studies im Gefolge von Stuart Hall und anderen, wenn sie von »conjuncture» und "conjunction" spricht. Um diese Filiation im Deutschen nachvollziehbar zu machen, werden in der Übersetzung auf das Lateinische zurückgehende Fremdwörter anstelle deutscher Wörter (»Konjunktion" statt "Verbindung») verwendet. Vgl. dazu auch Moritz Ege (2019): Cultural Studies als Konjunktur- und Konstellationsanalyse.

3 Um den Prozess der Untersuchung dieser Form der Intersektion auch formal zu beginnen, habe ich am Africana Studies Center der Cornell University ein Graduiertenseminar zu diesem Thema angeboten. Gespräche mit Rhoda Reddock halfen mir, das Thema aus unterschiedlichen Blickwinkeln anzugehen. Bei der Tagung der Caribbean Studies Association 2010 in San Andres hielt sie den Vortrag »The First Mrs. Garvey and Others: Pan-Africanism and Feminism in the Early $20^{\text {th }}$ Century British Colonial Caribbean«. Verschiedene Fassungen dieses Vortrags wurden auch an der Universität Legon in Ghana im April 2010 und bei der Callaloo-Konferenz "Black Movements: Poetics and Praxis« 2010 in Addis Abeba, Äthiopien vorgestellt. 
wärtigen Konzepte eines transnationalen Schwarzen Feminismus einordnen.

In der Berichterstattung über die panafrikanische Konferenz im Jahr 1900 werden die - »auf ihrem Gebiet allesamt bedeutenden" - Delegierten genannt, welche die USA, Kanada, Äthiopien, Haiti, Liberia, Sierra Leone, die damalige Goldküste und die meisten der damals britischen Westindischen Inseln vertraten. Zu der starken US-Delegation gehörten W.E. B. Du Bois, Miss Anna Jones (Kansas) und Mrs. Annie Cooper (d.h.: Anna Julia Cooper, Washington D. C.) (vgl. Adi und Sherwoood 2003). Anna Julia Cooper war als Autorin von $A$ Voice from the South (1892) in Erscheinung getreten, eines der ersten Bücher, das "Rasse» und Geschlecht theoretisch in einen Zusammenhang stellt. Dies war möglicherweise ein Grund für ihre Einladung zu der acht Jahre später stattfindenden Konferenz. Sie war auch eine Aktivistin für die Rechte von Schwarzen und Frauen. Aus der Geschichte des Panafrikanismus ist sie jedoch verschwunden (Guy-Sheftall 2007). Aufgrund der Ausrichtung ihrer Arbeit auf »Rasse« und Geschlecht konnte sie sich offensichtlich einem vermännlichten Panafrikanismus als einzigem Diskurs nicht anschließen. Bei einem der späteren, von Du Bois organisierten panafrikanischen Kongresse trug Jessie Redmon Fauset, die dort als Delegierte der National Organisation for the Advancement of Coloured Peoples vertreten war, Überlegungen mit dem Titel $॥$ Impressions of the $2^{\text {nd }}$ pan-African Congress" vor (vgl. Scott 2007, 247-252). Der fünfte panafrikanische Kongress 1927 in New York schließlich wurde auch von der Women's International League for Peace and Freedom gesponsert. ${ }^{4}$

Die frühen transnationalen Schwarzen Feministinnen verstanden die Konjunktion (das "und«) vermeintlich verschiedener politischer Positionen (Panafrikanismus und Feminismus) als zweifache Verortung, als eine Art Ko-Identifikation. Später wurde in der Debatte um »Panafrikanismus oder Kommunismus« das "Entweder-Oder» betont. ${ }^{5}$ Das Wort Feminismus erscheint allerdings in vielen dieser Zusammenstellungen gar nicht, obwohl es damals eine aktive Bewegung gab. Nach meiner Interpretation ist jedoch der deutlichste Beweis für eine Konjunktion in der politischen Ausrichtung der frühen Aktivistinnen zu finden. Ihre Aktionen gingen

4 Die Dokumentation »The Pan-African Congresses, 1900-1945 « auf der Webseite black past.org zeigt ein Foto von der dritten Konferenz 1921 in Brüssel, auf dem zwei Frauen auf der Bühne zu sehen sind.

5 George Padmore, ein wichtiger Theoretiker des Panafrikanismus, veröffentlichte 1956 das Buch "Panafrikanism or Communism". Es kann als exemplarisch gelten für das zu dieser Zeit aufkommende Denken in konkurrierenden politischen Modellen, wie es das »or« im Buchtitel anzeigt. 
nicht von einer separaten Schwarzen feministischen Bewegung aus, (die erst später entstand), sondern von einer Vorstellung der Gleichzeitigkeit verschiedener Formen politischer Unterdrückung und entsprechender politischer Positionen. So lieferte Honor Ford-Smith im Kontext der Debatte über die Garvey-Bewegung mit ihrem Beitrag zu Cathryn McKenzie, einer der ersten jamaikanischen Feministinnen, den Nachweis, dass "es historisch gesehen in der gesamten Dritten Welt eine enge Verbindung zwischen antikolonialen und feministischen Bewegungen gegeben hat" (Ford-Smith 1991, 73).

Die jüngste Manifestation einer Konjunktur, d.h. eines historisch gleichzeitigen Auftretens der beiden ideologischen Positionen, diesmal als Ergebnis langwieriger Arbeit, gab es interessanterweise 2005, als die Afrikanische Union im internationalen Recht für die Einführung von zwei neuen Instrumenten sorgte. Das Protokoll für die Rechte der Frauen in Afrika wurde gleichzeitig mit der offiziellen Anerkennung der Afrikanischen Diaspora verabschiedet. Rhoda Reddock macht in ihrer Einleitung zu $\mathrm{Fe}$ minist Africa 7 auf diese Koinzidenz aufmerksam, sie diskutiert dort die Bedeutung Afrikas für den Feminismus der Diaspora (Reddock 2007).

Ich behaupte, dass es schon immer Aktivistinnen gab und auch weiterhin geben wird, die konsistente Verbindungen zwischen afrikanischnationalen, afrikanisch-kommunistischen und feministischen Positionen herstellen. Im Laufe der Jahre setzte sich jedoch eine Tendenz durch, den Rahmen feministischer Analysen von panafrikanischen Positionen abzukoppeln und die panafrikanische Arbeit von Frauen damit herabzusetzen. Dies war mit einer Verengung des analytischen Blicks auf teilweise sehr enge lokale Kontexte verbunden, die zudem in kulturalistischer Manier ethnisch typisiert wurden. Ich verwende den Ausdruck "kulturalistisch" hier bewusst im Unterschied zu »kulturell«, um auf eine deterministische Tendenz der Konkretisierung von Kultur hinzuweisen, die dazu dient, diese von anderen gesellschaftlichen Strukturierungen zu unterscheiden oder gegen sie abzugrenzen (vgl. McClendon 2003).

Die wenigen Frauen in Afrika und in der afrikanischen Diaspora, die beständig derartige Konstellationen schufen oder die, wie Stuart Hall es formulierte, »die Konjunktur lebten« (Hall 2007, 272f.), sollen hier zumindest Erwähnung finden: Mabel Dove Danquah nahm am zweiten panafrikanischen Kongress teil. Ihr Ehemann Joseph Boakye Danquah, selbst ein bedeutender Panafrikanist, war einer jener afrikanischen Studenten, die Amy Ashwood Garvey in der Westafrikanischen Studentenunion in London gefördert hatte. Nach seiner Rückkehr nach Ghana half Joseph Danquah Ashwood, die Abstammung ihrer Familie und ihre 
Aschanti-Ursprünge zu rekonstruieren (Martin 2007). Mabel Dove Danquah ist mit ihren Kurzgeschichten wahrscheinlich nur in einigen afrikanischen Literaturzirkeln bekannt. Sie macht sich in ihren Erzählungen über alte, traditionsverhaftete Dorfvorsteher lustig, die sich in den Wirren ihrer exzessiven Ausübung der Polygamie und ihrem Verlangen nach neuen Frauen verstricken. In ghanaischen Kreisen ist sie auch als Panafrikanistin bekannt, aber sie verdiente es meiner Ansicht nach, eingehender als panafrikanische Feministin gewürdigt zu werden, die im transnationalen Schwarzen Feminismus verortet ist.

Über die Panafrikanistin Adelaide Casely Hayford, die 1903 den Panafrikanisten Joseph Ephraim Casely Hayford heiratete und dem Zweig der UNIA (United Negro Improvement Association) in Freetown vorstand, nachdem ihre Ehe mit Casely Hayford zerbrochen war, hat Beverly GuySheftall (2003) interessante Details zusammengetragen. Adelaide Hayford verbrachte zwei Jahre in den USA und recherchierte über Mädchenschulen. Sie verbündete sich mit US-Amerikanerinnen wie Nannie Burroughs und gründete später eine eigene Schule für Mädchen. ${ }^{6} 1927$ nahm sie am vierten Panafrikanischen Kongress in New York teil, der von W.E.B. DuBois organisiert wurde. Guy-Sheftall (2003) hat nachgewiesen, dass die in diesem Kontext engagierten Frauen häufig über Kontinente hinweg zusammenarbeiteten, internationale Freundschaften pflegten und Verbindung hielten.

Frauen aus dem linken Spektrum wie die Theoretikerin und Aktivistin der Kommunistischen Partei der USA, Claudia Jones, besuchten beim Weltkongress der Frauen 1963 in der UdSSR nachweislich die gleiche Zusammenkunft wie Mrs. Funmilayo Ransome-Kuti (Jones 1999, 110). ${ }^{7}$ Im Jahr 1964 halfen diese beiden in Japan eine Resolution gegen die Verbreitung und den Einsatz von Atom- und Wasserstoffwaffen durchzusetzen (Boyce Davies 2008). Sie gehörten der Women's International Democratic Federation (WIDF) an, die als linke Frauenorganisation galt. Dies ist auch ein Hinweis darauf, dass Ransome-Kuti, die in einem Broadway-Musical namens »Fela«, das von 2008 bis 2012 ständig auf dem Spielplan stand, als

6 Anm. d. Red.: Nannie Helen Burroughs war eine afroamerikanische Erzieherin, Rednerin, Religionsführerin, Bürgerrechtlerin, Feministin und Geschäftsfrau in den Vereinigten Staaten, die 1909 eine afroamerikanische Berufsschule für Frauen und Mädchen gründete.

7 Anm. d. Red.: Funmilayo Ransome-Kuti organisierte in den 1930er Jahren großangelegte Alphabetisierungskurse für Frauen im ländlichen Nigeria. 1949 gründete sie die Nigerian Women's Union (NWU), die demokratische Mitbestimmung, Wahlrecht für Frauen und proportionale Repräsentation von Frauen in den Gemeinderäten forderte, und setzte sich für die Autonomie Nigerias ein. 
Fela Kutis überirdische Mutter dargestellt wird, als klassenbewusste Frau wahrgenommen werden sollte (Odim und Mba 1997; Moore 1982; Mba 1982). ${ }^{8}$

Die Verbindung zwischen den beiden politischen Positionen lässt sich auch anhand einiger herausragender Persönlichkeiten der Diaspora veranschaulichen, die als Schwarze Feministinnen ein transnationales Leben führten. Ihre konkreten Aktionen und theoretischen Überlegungen sind voll von jenen Intersektionen, von denen oben die Rede war. Die bereits genannte Amy Ashwood Garvey, die erste Ehefrau von Marcus Garvey und Mitbegründerin der UNIA (United Negro Improvement Association), pflegte freundschaftliche Beziehungen sowohl zu Cyril Lionel Robert James als auch zu George Padmore. Öffentlich trat sie als Feministin in Erscheinung, während sie im Verborgenen ein Leben als aktive panafrikanische Strategin führte, wie Rhoda Reddock herausfand (Reddock 2014). ${ }^{9}$ Martin (2007) berichtet, dass Amy Ashwood Garvey auf Bitten von Jones für die erste Ausgabe der West Indian Gazette einen Aufsatz über Ghana verfasste. Dabei war Jones vor allem an "Amys Kommentaren zur Föderation und zur möglichen Unabhängigkeit der Karibischen Inseln« interessiert und an ihrer Einschätzung der Frage, "wie (falls überhaupt) die Ghanaer den neuen Bund im Rahmen von Nkrumahs Plan einer panafrikanischen Föderation beurteilen" (Martin 2007, 272)..$^{10}$

Die Verbindungen werden noch offensichtlicher, wenn Claudia Jones berichtet, dass ihr die in Reaktion auf Mussolinis Invasion in Abessinien entstandenen Arbeiten den Anstoß gegeben hätten, politisch aktiv zu werden. Die Analysen, die für sie zu einer Klärung beigetragen hatten, kamen von James, Padmore und Amy Ashwood, die alle drei zu dem Zeitpunkt Mitglieder der Organisation International African Friends of Abyssinia waren. Claudia kannte Amy offenbar bereits vor ihrer Auswanderung nach Lon-

8 Anm. d. Red.: Fela Kuti (1938-1997), einer der Söhne Funmilayos, gilt als Erfinder des Afrobeat.

9 Anm. d. Red.: C.L.R. James, geboren 1904 in Trinidad, wurde als Journalist, sozialistischer Theoretiker und Schriftsteller zunächst in England, dann in den USA bekannt. Während der McCarthy-Ära wurde er 1952 aus den USA ausgewiesen.

10 Anm. d. Red.: Kwame Nkrumah wurde 1957 der erste Präsident Ghanas. 1945 organisierte er den 5. Panafrikanischen Kongress in Manchester. Er gilt als einer der radikalen Vordenker von Panafrikanismus und Sozialismus in Afrika. 1950 wurde er im Rahmen politischer Unruhen gegen die britische Besatzung, die er mit initiiert hatte, inhaftiert. Die von ihm gegründete, sozialistische Convention Peoples Party CPP erlangte aufgrund ihrer Forderung nach umgehender Autonomie bei den Parlamentswahlen im Jahr 1951 die meisten Stimmen und die Briten entließen Nkruhmah aus der Haft. 1952 wählte ihn die gesetzgebende Versammlung zum Premierminister der Kronkolonie Goldküste. 
don. Aus allen Berichten geht hervor, dass die Appartmentanlage an der Edgecombe Road in Harlem, in der Claudia lebte, für die panafrikanischen Aktivitäten ihrer Bewohner bekannt war. Studenten wie Azikiwe ${ }^{11}$ kamen dorthin zu Besuch und sogar der junge Nkrumah soll als Besucher einer Reihe von Personen, wie Claudia Jones, Lorraine Hansberry und W.E.B. Du Bois, die dort lebten, gesehen worden sein. ${ }^{12}$

Eine weitere bedeutende Konjunktion zwischen Panafrikanismus und Feminismus zeigt sich in der Arbeit von Una Marson, die als feministische Aktivistin und Journalistin bekannt war, welche über die Erfahrungen Schwarzer Frauen sprach und schrieb. Sie unterstützte auch die Radiosendung Caribbean Voices beim BBC, in der die Karriere vieler heute bekannter Autorinnen und Autoren aus der Karibik begann (Jarrett-Macauley 2010). Sie schloss sich der Women's International League for Peace and Freedom und anderen Organisationen an. Die Konfrontation mit dem Rassismus in England schärfte ihre Wahrnehmung für die weltweiten Probleme der Ungleichheit zwischen den "Rassen«. Für unsere Diskussion ist freilich ihre Arbeit als Sekretärin bei der League of Coloured Peoples, der ersten von Schwarzen geleiteten politischen Organisation in England wichtiger. Sie wurde Sekretärin bei Haile Selassie und reiste 1936 mit ihm zum Völkerbund, um sich für die Befreiung Abessiniens einzusetzen. Als vermutlich erste bedeutende Dichterin und Bühnenautorin aus der Karibik hat sie sich einen Ruf als literarische Pionierin erworben, die sich mit der öffentlichen Darstellung Schwarzer Frauen und insbesondere mit ihrem Haar befasste. Mit Gedichten wie »Kinky Hair Blues« war sie dem zeitgenössischen Interesse weit voraus (Marson [1937] 2011). Wie panafrikanischen Zeugnissen $\mathrm{zu}$ entnehmen ist, hat sie sich neben ihrer Arbeit mit Haile Selassi schon für die Sache der Rastafari eingesetzt, als es noch nicht populär war, dies zu tun. Man weiß auch, dass sie sich an antikolonialen Kämpfen beteiligte und Norman Manley bei seiner Arbeit zur Durchsetzung der Unabhängigkeit Jamaikas von Großbritannien unterstützte.

Una Marson arbeitete ebenso wie Claudia Jones und Funmilayo Ransome-Kuti transnational mit Frauenrechtsorganisationen zusammen, etwa mit der feministischen International Alliance of Women. Allison Donnell ver-

11 Anm. d. Red.: Nnamdi Azikiwe war von 1963 bis 1966 erster Staatspräsident von Nigeria. Nach seiner akademischen Ausbildung in den USA gehörte er bereits in den 1930er Jahren zu jenen Impulsgebern der afrikanischen Unabhängigkeitsdiskussion, die dem Konzept westlicher Demokratie stets verbunden blieben. Zu seinen Schülern gehörte auch der junge Nkrumah.

12 Vgl. den Beitrag von Reddock in Feminist Africa 19 mit einer ausführlichen Erörterung von Amy Ashwood Garveys Rolle in der panafrikanischen Bewegung (Reddock 2014). 
tritt die interessante These, dass Marsons Frauenrechtspositionen schon gefestigt waren, ehe sie nach London ging (Donnell 2003, 125). Marson war sich im Klaren über die Gleichzeitigkeit verschiedener Formen von Unterdrückung, ihre Wahrnehmung panafrikanischer Bewegungen entstand parallel $\mathrm{zu}$ ihren übrigen Anliegen und dies ermöglichte ihr »die Dringlichkeit der Bekämpfung rassisch begründeter Hierarchien" (Donnell 2003, 117) zu begreifen. In einer solchen Konstellation lässt sich ohne Weiteres erkennen, dass mit der Entwicklung einer vollkommenen $\mathrm{Hu}-$ manität, in der die politische und wirtschaftliche Macht von allen geteilt wird, eine Reihe von Kämpfen gegen "alle Herrschaftssysteme" verbunden ist. Diese Konstellation erinnert in mancher Hinsicht auch an die ersten Ausdrucksformen der zweiten Welle Schwarzer feministischer Politik in den USA (auch in den 1980ern), wo eine Form des Feminismus artikuliert wurde, der die Kategorien Klasse und "Rasse« ebenso berücksichtigt wie die Kategorie Geschlecht (vgl. die Einleitung in Hull et al. 1982).

\section{Die Grenzen "kulturalistischer" Analysen afrikanischer Frauenrechte}

Je näher wir dem Ende des 20. Jahrhunderts kamen, desto mehr beherrschte eine Vorstellung von Politik als Kulturpolitik den Diskurs sowohl im Panafrikanismus als auch im Feminismus, mit der Tendenz, die Kultur zu feiern und das politische System zu übergehen. Dies war ein notwendiger Schritt, da die Kultur einer der entscheidenden Hebel zur Selbstermächtigung ehemals kolonisierter Gruppen und Gemeinschaften ist (Fanon 1961). ${ }^{13}$ Allerdings vermittelt ein solcher Ansatz von Kultur, ohne Analysen, die eine Verbindung zu anderen, soziopolitischen und historischen Systemen herstellen, eine kulturalistische Orientierung, die verschiedene miteinander verbundene Formen der Unterdrückung verdeckt. Damit hat es meiner Meinung nach auch zu tun, dass »Klasse« als entscheidende Variable aus den neueren Untersuchungen afrikanischer Geschlechtersysteme verschwunden ist.

Der Euro-Feminismus wurde als übergeordnete Position bereits von verschiedenen Seiten aufgrund seiner Annahme kritisiert, dass alle Frauen

13 Fanon (1961) hat eine der bekanntesten Formulierungen für die Bedeutung nationaler Kultur ausgearbeitet, die allerdings allgemeinen Charakter hat, Frauen nicht als besondere Gruppe berücksichtigt und vor allem die frühen Formen Schwarzer nationalistischer Politik in den Blick nimmt. Die Kritik an seinem Umgang mit der Kategorie Geschlecht ist bekannt (z. B. Loomba 1998; Mama 1995). 
Unterdrückung in ähnlicher Weise erlebten und ihr Zugang zur Macht gleich verlaufe. Europäisch-amerikanische Kulturformationen gehen selbst im Rahmen des feministischen Diskurses, der sich innerhalb dieser Formation entwickelt hat, von einer bestimmten Universalität wie auch von einem impliziten kolonialen Privileg aus. Deshalb musste erst ein afrikanischer Feminismus von afrikanischen Denkerinnen wie etwa Filomina Steady aus Sierra Leone hervorgebracht werden. Allerdings waren diese ersten Äußerungen sehr stark auf die Natur-Kultur-Dichotomie ausgerichtet und essentialistisch, obwohl gesellschaftliche Veränderungen gefordert wurden (Steady 1981; Boyce Davies / Graves 1986).

Auf institutioneller Ebene trat die Definition von Geschlechtersystemen für Frauen in afrikanischen und karibischen Kontexten an die Stelle kritischer feministischer Studien. Dabei spielte die Verbindung von Genderund Entwicklungsforschung und die Einrichtung entsprechender Abteilungen und Institute eine wichtige Rolle, wie dies etwa an der University of the West-Indies der Fall war. Die Zentren für Gender und Entwicklung verbanden sich mit Aktivitäten von Nicht-Regierungsorganisationen, die sich im Anschluss an verschiedene UN-Mandate im Rahmen des Entwicklungsprogramms für Frauenrechte einsetzten. Auf wissenschaftlicher Ebene begann man mit einer Vielzahl von Konzepten zur Stellung von Frauen im afrikanischen Kontext auch den Begriff der Entwicklung umzuarbeiten. Molara Ogundipe (1984) war sich dieser Verknüpfung bewusst und gab ihrer Untersuchung der sozioökonomischen Wirklichkeit von Frauen den Titel "Eine andere Entwicklung«. Ganz bewusst verwendete sie die maoistische Metapher des "Berges«, um das Ausmaß der vielen Arten von Unterdrückung zu kennzeichnen: Kolonialismus, Tradition, neokoloniale Realitäten der Unterentwicklung und Armut, männliche Macht und "Rasse", die sie entlang klassistischer und rassistischer Kriterien unterteilte. In ihrer Diskussion der Unterdrückungsformen stellte Ogundipe eine Argumentationslinie vor, in der die Lebenswelten afrikanischer Frauen zwar auf der Grundlage eines Klassen- und Geschlechtersystems analysiert werden, zugleich aber auch zum Ausdruck kommt, wie diese Realitäten zu kulturellen Praktiken werden.

Ein anderer, eher afrozentrischer Wissenschaftsansatz griff die häufig gegen den Feminismus als westlichen Import geäußerte Kritik auf, indem er sich selbst im Kontext besonderer ethnischer/kultureller Paradigmen verortete. So demonstrierte Ifi Amadiume (1987) an ihrer Igbo-Gemeinschaft, dass darin verschiedene Geschlechter- bzw. Machtdynamiken wirksam sind, die es den Frauen, wenn sie ökonomische Vorrechte erlangt haben, erlauben, einige der Rechte wahrzunehmen, die sonst Män- 
nern vorbehalten sind. Damit würdigte sie nicht nur die Bedeutung wirtschaftlicher Aktivitäten von Frauen als wesentliche Grundlage von Macht, sondern wendete sich zugleich gegen die Vorstellung, das westliche Geschlechtersystem sei als das fortschrittlichste Modell nachahmenswert. In ihrem nächsten Buch Re-Inventing Africa: Matriarchy, Religion and Culture arbeitete Amadiume (1998) ihr Vorhaben zur Wiedererweckung unterrepräsentierter afrikanischer Epistemologien weiter aus. Amadiume (1987), die im Bereich der afrikanischen Gender Studies maßgeblichen Einfluss hatte, lieferte das Vorbild dafür, wie mit den spezifischen Beispielen besonderer ethnischer Gruppen übergreifende Definitionen einer vereinheitlichenden westlichen Geschlechtervorstellung anzufechten sind. Einige westliche Feministinnen begannen umgekehrt, Amadiumes Arbeit dahingehend zu verallgemeinern, dass sie deren Ausführungen auf die gesamte Gesellschaft der Igbo und darüber hinaus auf kulturelle Vorstellungen von Heirat und Sexualität in ganz Afrika bezogen. Amadiume hat in späteren Arbeiten ihre Position weiter ausgearbeitet und sich selbst dabei als eine Frau beschrieben, die sich kritisch mit der Entwicklungsdoktrin auseinandersetzt, um Forderungen nach Gleichbehandlung und Geschlechterdemokratisierung Anerkennung zu verschaffen. Genau genommen besteht ihr Vorschlag darin, durch die "kollektivistische, humanistische Kultur des traditionellen afrikanischen matriarchalen Erbes« (Amadiume 2000, 302) Wiedergutmachung zu erreichen. So gesehen, bewegt sich Amadiumes Werk in zwei verschiedene Richtungen: Kritik an den westlichen Annahmen über Geschlechterverhältnisse bei gleichzeitigem Verweis von Frauen an einen traditionellen Ort im kulturellen afrikanischen Erbe.

In diesem Zusammenhang ist auch der Ansatz von Oyeronke Oyewumi (1997) zu nennen, in dem die Volksgruppe der Oyo-Yoruba, der die Autorin angehört, für die Ausformulierung eines ethno-kulturellen Arguments und eine umfassende Kritik herangezogen wird. Oyewumi (1997) erweitert das kulturelle Argument, indem sie die These aufstellt, dass nach ihrer Interpretation in der Yoruba-Gesellschaft »Geschlecht" gar nicht existierte, sondern eigentlich eine vom Westen aufgezwungene Kategorie sei. Dazu werden in The Invention of Women (Oweyumi 1997) vier Argumente entfaltet und in Varianten wiederholt, so dass die folgenden Schlussfolgerungen unmissverständlich sind:

1. Afrika-Studien sind westlich dominiert und arbeiten mit westlichen Kategorien, die auf afrikanische Realitäten angewendet werden.

2. Westliche, koloniale Dominanzstrukturen (Wissen, Ökonomie, Kulturleben, Sprach- und Geschlechtskategorien), Formen des Zugangs zum Geistesleben und deren Anwendungen auf Menschen prägen die 
Wissenschaft, mit dem Ergebnis, dass eine ganze Generation afrikanischer Wissenschaftler und Wissenschaftlerinnen westliche Annahmen über das Patriarchat nur allzu leichtfertig auf afrikanische Gesellschaften überträgt.

3. Die westliche feministische Wissenschaft geht von einer universellen Unterordnung der Frauen aus und nimmt an, dass Frauen eine Gruppe ohne weitere innere Differenzierung darstellen; überdies sind diese westlichen feministischen Wissenschaftlerinnen von der Vorstellung ausgegangen, alle Kulturen beruhten auf Geschlechterverhältnissen.

4. Das zentrale Argument aber, das die drei zuvor genannten vorbereiten, ist die kühne und provokative Behauptung, dass die Kultur der Yoruba frei von Geschlechtereinteilungen sei und dass jede Anwendung von Kategorien des Geschlechts auf die Yoruba-Gesellschaft ihr ein System auferlege, das vor der Kolonialisierung nicht bestanden habe.

Aus meiner Sicht erwecken diese Behauptungen den Anschein, Oweyumi konstruiere eine undifferenzierte und statische Yoruba-Gesellschaft oder vielleicht auch eine essentialistische Yoruba-Identität. Ein Großteil ihrer Analyse beruht auf der Untersuchung von Sprache, und sie kommt zu der Schlussfolgerung, dass Yoruba eine Sprache ohne Geschlechtskategorien sei (Oweyumi 1997, 175). Der Versuch, einen Weg jenseits der gängigen analytischen Kategorien zu finden, ist ein lobenswertes Ansinnen. Meiner Ansicht nach hat die Unerschrockenheit des Buches viel mit dem zu tun, was in Ibadan bolekaja (es auf dem Boden der Tatsachen miteinander ausfechten) genannt wurde: eine besondere Art der intellektuellen Arbeit. ${ }^{14}$ Damit gelingt es Oyewumi, eine Schwachstelle der westlichen feministischen Arbeit auszumachen und eine konkurrierende These in die Debatte einzubringen: "Die sozialen Kategorien der Yoruba beruhten nicht auf anatomischen Unterschieden" (Oweyumi 1997, 176).

Doch die Annahme, in der Kultur der Yoruba habe es keine Geschlechtskategorien gegeben, ist analytisch betrachtet widersinnig, sie verstößt gegen eine Grundlogik der menschlichen Natur, nämlich gegen die Art, wie Gesellschaften bereits vor dem Kolonialismus und auch danach stratifiziert wurden. Auch unterstellt Oweyumi die Einzigartigkeit der Yoruba-Kultur, ohne sich auf eine gründliche historische und soziologi-

14 Oyewumi, die in den späten 1970er Jahren Studentin an der Universität von Ibadan war, als ich dort in den höheren Semestern studierte, tendierte zu diesem Ansatz, der wissenschaftliche Ideen als einander widerstreitende Positionen sah. Der von ihr verwendete Bokelaja-Ansatz war damals sehr beliebt und wurde von einigen Intellektuellen, etwa dem jungen Professor Abiodun Jeyifo, gefördert. 
sche Erforschung vorkolonialer Organisationssysteme der Yoruba stützen zu können. In diesem Zusammenhang bietet Bibi Bakare-Yusufs (2004) Analyse der Arbeit Oyewumis eine wichtige Neubewertung der kulturalistischen Thesen. Sie kommt zu dem Ergebnis, dass dieser analytische Ansatz die Überschneidungen zwischen Klassen- und ökonomischen Unterschieden häufig auslöscht, während die Überschneidungen zwischen Alters- und anderen Machtbeziehungen, einschließlich der Geschlechterverhältnisse berücksichtigt werden.

Nach Oyewumi ist die "Frauenfrage " eine westliche Frage und kein geeignetes Instrument zur Beurteilung afrikanischer Gesellschaften. Doch kann man zwar behaupten, dass die »Frauenfrage« von Beginn an westlich war, sollte aber meines Erachtens berücksichtigen, dass diese Benennung aus der sozialistischen Politik Clara Zetkins (1934) hervorging, die darüber mit Lenin debattiert hatte. Sie wollte das Problem der Frauen auf die Tagesordnung setzen, als es in den grundlegenden Klassenanalysen noch nicht einmal erwähnt wurde (Boyce Davies 2007). Die "Frauenfrage« entstammt einer früheren Kritik an der Tilgung der Beiträge von Frauen als denkenden und handelnden Subjekten aus der Geschichte verschiedener Systeme. Oyewunmi, die in ihrer Analyse die Ethnie zum wichtigsten Unterscheidungsmerkmal macht, bietet zugleich eine Festschreibung des Nativismus an. Afrikanische Geschlechtersysteme werden auf diese Weise für die soziopolitischen und klassenbewussten Absichten der frühen afrikanischen Feministinnen, welche die "Konjunktion lebten«, gesperrt.

Der Gedanke einer "Wiederherstellung lokaler Epistemologien" ist allerdings verlockend und in diesem Zusammenhang könnte die Diskussion über Iyalode zur Entwicklung eines Vorbilds weiblicher Kraft, Stärke und Führung beitragen, das zur Beschreibung der Arbeit und Bedeutung von Frauen wie Funmilayo Ransome-Kuti taugt. ${ }^{15}$ Ransome-Kuti war, wie gesagt, eine eigenständige feministische Aktivistin mit internationalen Verbindungen zu linken Frauengruppen. Sie ging in ihrem Ansatz, in Nigeria Frauenrechte durchzusetzen, strikt von Geschlechter- und Klassenzugehörigkeiten aus. In Oyewumis Arbeit hingegen werden einige Probleme armer Frauen und Männer aus der Arbeiterklasse zu undifferenzierten Problemen der Yoruba als Volk. Es wird von einer klassenlosen Position ausgegangen, obwohl "Klasse« als analytische Kategorie nicht verwendet wird und auch niemals den Rahmen für die Analyse abgibt.

15 Anm. d. Red.: Iyalode ist eine weibliche Führungsfigur bei den Yoruba, die aufgrund ihrer Leistungen in wirtschaftlichen und politischen Angelegenheiten eine herausgehobene Stellung einnahm. 
Auf diesem Gebiet bedarf es dringend weiterer Forschung, und selbstverständlich sollte sie sich auf einen Geschlechter- und Klassenansatz stützen.

\section{Klassen- und Geschlechter-Systeme in der feministischen Analyse}

Die Konjunktion von linkem Gedankengut und Feminismus wird in der neueren Literatur zum Thema afrikanische Frauen weitgehend vernachlässigt und vielleicht ist gerade dies der Aspekt, der dem von mir kritisierten kulturalistischen Feminismus fehlt. Es ist offensichtlich, dass der Erforschung von Klassen- und Geschlechtersystemen als elementarem Bestandteil afrikanischer Gesellschaften deutlich mehr Aufmerksamkeit zuteil werden muss. So zeigt eine Auswertung der letzten zehn Jahrgänge der Zeitschrift Feminist Africa, dass dem Thema Kultur eine Reihe von Heften gewidmet war (Heft 2, 5, 6, 16, 17), einige sich mit Wissenschaftspolitik und der Rolle der Universitäten (Heft 1, 8, 9) sowie verschiedenen Formen des Aktivismus (Heft 4, 5, 8, 11, 18) beschäftigten und eine Ausgabe die Diaspora in den Blick nahm (Heft 7). Vorbildlich ist jedoch das Sonderheft Land and Labour and Gendered Livelihoods (Heft 12), weil es die Diskussion über Frauenarbeit in den verschiedenen Produktionsweisen fortführt. ${ }^{16}$

Da es bereits gute sozialwissenschaftliche Untersuchungen von afrikanischen Wissenschaftlerinnen gibt, die sich mit den Komplexitäten der Klassen- und Geschlechterdynamik befassen und insbesondere die gegenwärtigen Lebensumstände von Frauen analysieren (z.B. Ampofo 2007), kann es sein, dass die neue Popularität kulturwissenschaftlicher Studien die politische Ökonomie in den Hintergrund gedrängt hat, in der Fragen der Verschränkung von Klasse und Geschlecht unausweichlich sind. Es mag deshalb nützlich sein, ohne Rücksicht auf das Kommen und Gehen diskursiver Trends eine Forschungsfrage aufzuwerfen, die von anhaltender Bedeutung ist: Wie sind die Klassen- und Geschlechterverhältnisse in afrikanischen Gesellschaften beschaffen, die arme Frauen unter den gegenwärtigen Wirtschaftssystemen benachteiligen?

Angesichts einseitiger Klassenanalysen in früheren linken Untersuchungen äußerte die feministische Historikerin Joan Scott sich kritisch, dass

16 Feminist Africa 12 (2009) hat eine Reihe von Aufsätzen über verschiedene Orte von Ghana bis Kenia veröffentlicht, in denen der geschlechtsspezifischen Politik agrarischer Produktion in Farmhaushalten besondere Aufmerksamkeit zukommt. 
auch Geschlecht eine grundlegende Kategorie der Strukturierung von Machtbeziehungen sei. Im »Marxismus wurde das soziale Geschlecht [...] als Nebenprodukt der sich verändernden ökonomischen Strukturen verstanden [...] hatte jedoch nie einen unabhängigen analytischen Status" (Scott 1994, 42). Sylvia Wynter diskutiert die politischen Auswirkungen einer Perspektivenverschiebung und hält fest, dass "Frauen im Anschluss an die 1960er Jahre aufhörten, das marxistische Denken wie ein Echo zu wiederholen und die Frauenfrage neu definierten" so dass das Problem nunmehr sie selbst und ihre Anliegen betraf, statt wie zuvor als Teilbereich dessen verstanden zu werden, was man die Frage der Arbeit nennen könnte. Wynter meint aber auch, dass es die »vielfältigen mit diesen Fragen verbundenen Bewegungen [waren], die sich am nachdrücklichsten in konkreten politischen und sozialen Kämpfen auf dem gesamten Erdball« niedergeschlagen haben (Wynter 2003, 312). Die Bandbreite und Vielschichtigkeit sozialer Bewegungen hatten marxistische Feministinnen schon vor den 1960er Jahren aufgezeigt. Und sogar noch früher - in den 1940er und 1950er Jahren - beschäftigten sich intellektuelle Frauen beziehungsweise Aktivistinnen wie Claudia Jones bereits mit dem sozioökonomischen Status Schwarzer Frauen aus der Arbeiterklasse (Boyce Davies 2007). Heute gibt es im wissenschaftlichen Diskurs deshalb keine statische und eindimensionale Bedeutung von Geschlecht mehr, weil sich Schwarze feministische Wissenschaftlerinnen seit langem darum bemühen, Macht in ihrer intersektionalen Wirkungsweise zu analysieren. Die Frage, wie Geschlecht und Klasse sich im afrikanischen Kontext verbinden und überschneiden, ist noch immer ein maßgebliches Analyseinstrument.

Mit seiner Hilfe können wir zu den konkreten Lebensrealitäten derjenigen vordringen, die in einer vergeschlechtlichten, klassistischen und rassialisierten Welt leben, die mehr oder weniger alles bestimmt, z. B. welche Löhne die afrikanischen Frauen bekommen, ob sie zu essen haben oder ob Seilschaften korrupter Beamter auf lokaler und internationaler Ebene ihr Öl fördern und sie enteignen. Die Frauen, die die Chevron- und ExxonÖlgesellschaften in Nigeria besetzten, können dies bezeugen (vgl. Ekine 2008). ${ }^{17}$ Dass es Frauen waren, die diese Aktion durchführten, ist signifikant. Zwar mag der Gewinn der Aktion im Hinblick auf die allgemeine Lage als gering angesehen werden, aber es gelang den Frauen immerhin, kleinere Zugeständnisse zu erwirken, wie Mikrokredite für Investitionen und Zusagen zur Förderung der Ausbildung und Beschäftigung ihrer

17 »Nigerian Women Force Oil Company to Take Corporate Responsibility« [http:// gbgm-umc.org/umw/legislative/oilcompanies.html] abgerufen am 12. August 2014. 
Kinder. Ein an Klassen- und Geschlechterverhältnissen orientierter Aktivismus ist also angesichts der gesellschaftlichen und wirtschaftlichen $\mathrm{Ge}$ gebenheiten der Gegenwart immer noch von Bedeutung. Dies gilt auch dann, wenn ältere feministische Strategien angewendet werden, wie der Einsatz des entblößten weiblichen Körpers während der Aba-Frauenaufstände von 1926 als ein Mittel des Protests, das im Friedensaktivismus liberianischer Frauen und anderswo wieder aufgegriffen wurde.

Untersuchungen, in denen die Kategorien Geschlecht, Klasse und »Rasse« miteinander verknüpft sind, gewinnen in der Forschung wieder an Ansehen. Dayo F. Gore (2011) zeigt, dass eine ganze Generation international geprägter Aktivistinnen als Bindeglied zu jenen feministischen Klassen- und Geschlechteranalysen fungierte, die am Ursprung des von Claudia Jones popularisierten kategorialen Rahmens von Klasse, "Rasse" und Geschlecht stehen. Diese Aktivistinnen machten wiederholt die hochgradige Ausbeutung von Frauen zum Thema, zumal diese insbesondere die Stellung von schwarzen Frauen in der Gesellschaft betrifft. Andere Anliegen waren die Auswirkungen des Krieges auf das Leben von Frauen, die Notwendigkeit, eine allgemeine Form der Organisation jenseits spezieller Belange zu finden, aber auch die Gründung von Frauen-Friedensorganisationen und die Entwicklung internationaler Verbindungen unter Frauen. Der Umstand, dass Frauen die Hälfte der Weltbevölkerung ausmachen, ist heute noch immer so relevant wie er es damals schon war (vgl. auch Boyce Davies 2011).

\section{Transnationaler Schwarzer Feminismus oder panafrikanischer Feminismus?}

Heute bringt der sogenannte transnationale Schwarze Feminismus die Logik der Überschneidung von Panafrikanismus und Feminismus auf den Begriff. Julia Sudbury schreibt:

[...] der transnationale Feminismus ist Teil eines großen anti-imperialistischen, anti-kapitalistischen Projekts [...] Transnationale feministische Praktiken laufen parallel zu der Arbeit antirassistischer Feministinnen, insofern als beide Gruppen die Überschneidungen von Geschlecht mit "Rasse", Klasse und Sexualität theoretisieren [...] diese Herangehensweise konzentriert sich auf Verbindungen, die aus transnationalen Netzwerken wirtschaftlicher und sozialer Beziehungen hervorgehen (Sudbury 2005, xviii). 
Jacqui Alexander (2005) hat die diskursiven Positionen des transnationalen schwarzen Feminismus detailliert zusammengetragen und zieht daraus den Schluss:

Wenn wir anerkennen, dass die Bedeutung des Nationalstaats für manche Personen schwerer wiegt als für andere, müssen wir auch zugestehen, dass die Grenzen des Nationalstaats weder hermetisch geschlossen sind, noch besondere Epistemologien begründen. Wir müssen mit unseren Vorhaben, Erkenntnisse zu gewinnen, über die staatlich konstruierten Grenzen hinausgehen, um kategoriale Rahmen entwickeln zu können, die sowohl intersubjektiv, komparativ und relational als auch historisch spezifisch und situativ fundiert sind. Und weil Fragmentierung sowohl materiell als auch metaphysisch, sowohl epistemisch als auch ontologisch ist, sollten diese Rahmen auch für die Schwachstellen von Macht und Überlegenheit und deren psychische Ökonomien als Bestandteile eines explizit politischen Projekts sensibel sein (Alexander 2005, 253 f.).

Ein wachsendes Korpus wissenschaftlicher Literatur Schwarzer und Farbiger Frauen befasst sich systematisch mit den Besonderheiten des Lebens von Frauen an unzähligen Orten und zeigt auf, was die Besonderheiten von Geschlecht, Sexualität, Ethnie, Klasse etc. bedeuten, wenn sie durch eine andere Brille betrachtet oder zumindest der Fixierung durch den westlichen Blick entzogen werden. Vor einiger Zeit hat Chandra Mohanty eine wichtige Diskussion über die Dekolonialisierung, ihre Anwendung auf feministische Bewegungen und eine zeitgenössische Rechtfertigung für transnationale feministische Politik in Gang gesetzt. Dabei stieß sie auf neue Herausforderungen: "zwar mögen feministische Ideen und Bewegungen weiter gewachsen und gereift sein, der Rückschlag und die Kampfansagen gegen den Feminismus haben jedoch ebenfalls exponentiell zugenommen« (Mohanty 2003, 2f.).

In meiner Auseinandersetzung mit der Literatur des transnationalen Schwarzen Feminismus (Boyce Davies 2008; 2011) behaupte ich, dass die Arbeit von Claudia Jones in vieler Hinsicht das vorwegnimmt, was wir heute als transnationalen Schwarzen Feminismus oder antiimperialistischen Schwarzen Feminismus bezeichnen. Ich habe festgestellt, dass in der Arbeit transnationaler Schwarzer Feministinnen anerkannt wird, dass unsere heutigen geografischen Verortungen Produkte mannigfaltiger historischer Prozesse sind, über die wir häufig gar keine Kontrolle hatten. Durch sie wurden wir weltweit zu Subjekten [Unterworfenen] verschiedener "Nationalstaaten", die mit Individuen ähnlicher oder anderer Herkunft interagieren müssen. Diese Verschiebungen sind das Ergebnis 
einiger abscheulicher Prozesse: Kriege, Kolonialismus, Versklavung, Holocaust, Lagerhaft, Enteignung und Genozid sowie diverse Unternehmungen unter dem Vorzeichen der Moderne. Claudia Jones hat bereits in den 1940er Jahren ausdrücklich auf diesen Zusammenhang hingewiesen, als sie zur Internationalisierung der Frauen-Friedensarbeit aufrief. Darunter verstand sie die Bildung von Koalitionen, die jenseits der männlichen Kriegslogik an einer Verbesserung der Lebensumstände arbeiten. Das Vorbild von Claudia Jones weist uns auf eine Reihe von Arbeitstätigkeiten hin, in der sich politische und geistige Arbeit miteinander verbinden.

In eine Analyse, die Feminismus und Antiimperialismus zusammenbringt, geht ein Minimum an Metadiskursen von unterschiedlichen Standpunkten ein. Der transnationale Feminismus geht von zwei Grundannahmen aus: Erstens, dass die Arbeit über Grenzen und Kulturen hinweg ein wesentliches Merkmal unserer heutigen Welt ist. Zweitens, dass unsere spezifischen Verortungen und Identitäten Bestandteil unserer Analysen sein müssen. Wenn wir dies berücksichtigen, muss jede kulturelle und politische Arbeit, die den festgefahrenen Gleisen und spezifisch imperialistischen Anrufungen entkommen will, ihren jeweiligen Standort ausweisen, ihre Besonderheit deutlich machen und zugleich bereit sein, das Vorhandensein anderer Kulturen und anderer politischer Realitäten anzuerkennen.

Für die Arbeit in transnationalen feministischen Zusammenhängen ist es vielleicht entscheidend, anzuerkennen, dass die Nationalstaaten, in denen wir als Subjekte leben, selbst aus spezifischen politischen Imperativen und historischen Prozessen hervorgegangen sind und deshalb versuchen, mannigfaltige Bevölkerungsgruppen den Wechselfällen ihrer nationalstaatlichen Unternehmen willkürlich zu unterwerfen. Wenn solche $\mathrm{Na}$ tionalstaaten eine Vormachtstellung erreichen, wie es in den USA der Fall war, werden unabsichtlich sogar innenpolitisch untergeordnete Identitäten in die Vormachtstellung einbezogen. Zahllose Menschen, die durch globale ökonomische Prozesse vertrieben und verdrängt werden, müssen sich auf die Dauer damit abfinden, emotional und physisch in verschiedenen Räumen zu leben. Sie mögen sich in den gemeinhin als "Diaspora" bezeichneten Raum begeben, ein Ort, der sich der Zentrierung widersetzt, auch wenn er für Sehnsucht, Heimat und einen Mythos vom Ursprung einsteht. Es gibt auch manche, die außerhalb der Diaspora bleiben, und manche, die in mehreren, sich überschneidenden oder überlappenden Welten der Diaspora leben.

Da progressives feministisches Denken im Kontext der Globalisierung nur transnational sein kann, und da es eine transnationale Version des 
Schwarzen Feminismus gibt, die vom Panafrikanismus beeinflusst wurde, haben wir die Möglichkeit, auf Modelle zurückzugreifen, die es in der politischen und intellektuellen Arbeit früher schon gab. Für uns, die wir in dieser Phase der kapitalistischen Globalisierung und angesichts extrem wachsender Unterschiede zwischen Menschen mit und ohne Zugang zu Macht und Ressourcen arbeiten, bedarf es einer weiterentwickelten Kritik am Imperialismus in all seinen Formen. Dennoch ist es wichtig, festzuhalten, dass schon die früheren Schwarzen Kommunistinnen eine Form von transnationalem Schwarzem Feminismus praktizierten. So lassen sich in der international ausgerichteten kommunistischen / feministischen Arbeit manche Kontinuitäten ausmachen. Sie ermöglichten es den Frauen, ihre Widerstandsfähigkeit und ihre Positionen zu behaupten, auch dort, wo verschiedene Standpunkte sich überschnitten: Anti-Imperialismus und Dekolonialisierung, Kämpfe für die Rechte von Arbeiterinnen, Kritik an der Aneignung der Arbeit Schwarzer Frauen, Kampfansagen gegen den einheimischen und den internationalen Rassismus und ihre Verbindung mit dem Kolonialismus. Aus diesen Überschneidungen ging ein transnationaler Schwarzer Feminismus hervor, der uns heute noch Vorbild sein kann. Wenn es uns gelänge, diese anhaltenden Bemühungen in gegenwärtige Artikulationen panafrikanisch-feministischer Politiken zu integrieren, könnten sie große Wirkung entfalten.

Aus dem Englischen von Regine Othmer

\section{Literatur}

Adi, Hakim/Sherwood, Marlka (Hrsg.) (2003): Pan African History. Political Figures from Africa and the Diaspora since 1787. London and New York.

Alexander, Jacqui (2005): Pedagogies of the Crossing. Durham, IMC.

Amadiume, Ifi (1987): Male Daughters, Female Husbands: Gender and Sex in an African Society. London.

Amadiume, Ifi (1998): Re-Inventing Africa: Matriarchy, Religion and Culture. London.

Amadiume, Ifi (2000): Daughters of the Goddess, Daughters of Imperialism: African Women Struggle for Culture, Power and Democracy. London.

Ampofo, Akosua Adomako (2007): My Cocoa Is Between My Legs: Sex as Work among Ghanaian Women/Work as a Duty and as a Joy: Understanding the Role of Work in the Lives of Ghanaian Female Traders of Global Consumer Items. In: Harley, Sharon (Hrsg): Women's Labour in the Global Economy. Speaking in Multiple Voices. New Brunswick, NJ, 182-220.

Bakare-Yusuf, Bibi (2004): Yorubas Don't Do Gender: A Critical Review of Oyeronke Oyewumi's The Invention of Women: Making an African Sense of Western Gender 
Discourses. In: Arnfred, Signe (Hrsg): African Gender Scholarship: Concepts, Methodologies and Paradigms. Dakar, 61-81.

Boyce Davies, Carole (2007): Left of Karl Marx. The Political Life of Black Communist Claudia Jones. Durham, NC.

Boyce Davies, Carole (2008): Encyclopedia of the African Diaspora: Origins, Experiences, and Culture, Band 1. Santa Barbara.

Boyce Davies, Carole (Hrsg.) (2011): Claudia Jones: Beyond Containment. Banbury.

Boyce Davies, Carole/ Graves, Anne Adams (1986): Ngambika: Studies of Women in African Literature. Trenton, NJ.

Donnell, Allison (2003): Una Marson: Feminism Anti-Colonialism and a Forgotten Fight for Freedom. In: Schwarz, Bill (Hrsg.): West Indian Intellectuals in Britain. Manchester, $114-131$.

Dove Danquah, Mabel (1994) Anticipation. In: Bruner, Charlotte (Hrsg.): Unwinding Threads. Oxford.

Ege, Moritz (2019): Cultural Studies als Konjunktur- und Konstellationsanalyse. In: Zeitschrift für Kulturwissenschaften Nr. 2: Kultur und Bildung - kulturelle Bildung?

Ekine, Sokari. 2008. Women's responses to state violence in the Niger Delta. In: Feminist Africa Issue 10/2008: Militarism, Conflict and Women's Activism, 67-83

Fanon, Frantz (1961): The Wretched of the Earth. New York.

Fanon, Frantz (1966): Die Verdammten dieser Erde. Aus dem Französischen von Traugott König. Frankfurt a.M.

Feminist Africa. Issue 12 (2009): Land, Labour and Gendered Livelihoods.

Ford-Smith, Honor (1991): Women and the Garvey Movement in Jamaica. In: Lewis, Rupert Bryan, Patrick (Hrsg.): Garvey. His Work and Impact. Trenton, NJ, 73-83.

Gore, Dayo F. (2011): Radicalism at the Crossroads. African American Women Activists in the Cold War. New York and London.

Guy-Sheftall, Beverly (2003): Speaking for Ourselves. Feminisms in the African Diaspora. In Boyce Davies, Carole/Gadsby, Meredith/Peterson, Charles/Williams, Henrietta (Hrgs.): Decolonizing the Academy: African Diaspora Studies. Trenton, NJ, $27-43$.

Guy-Sheftall, Beverly (2007): Foreword. In: May, Vivian M.: Anna Julia Cooper, Visionary Black Feminist: A Critical Introduction. London.

Hall, Stuart (2007): Epilogue: Through the Prism of an Intellectual Life. In: Meeks, Brian / Hall, Stuart: Caribbean Reasonings. Culture, Politics, Race and Diaspora: The Thought of Stuart Hall. London, 272-273.

Hull, Gloria T./Bell-Scott, Patricia/Smith, Barbara (1982): All the Women are White, All the Blacks are Men, But some of Us are Brave. New York.

Jarrett-Macauley, Delia (2010): The Life of Una Marson, 1905-1965. Manchester.

Loomba, Ania (1998): Colonialism / Postcolonialism. London and New York.

Mama, Amina (1995): Beyond the Masks: Race, Gender, and Subjectivity. London and New York.

Marson, Una (2011): Selected Poems. Edited by Alison Donnell. Leeds, UK.

Martin, Tony (2007): Amy Ashwood Garvey: Pan-Africanist, Feminist and Mrs. Marcus Garvey No. 1 or a Tale of Two Amies. Wellesley, MA.

Mba, Nina Emma (1982): Nigerian Women Mobilized: Women's Political Activity in Southern Nigeria, 1900-1965. Berkeley, CA

McClendon, John III (2003): From Cultural Criticism: Philosophical Idealism, Paradigmatic Illusions and the Politics of Identity. In: Boyce Davies, Carole/Gadsby, Mere- 
dith / Peterson, Charles / Williams, Henrietta (Hrsg): Decolonizing the Academy: African Diaspora Studies. Trenton, NJ, 3-26.

Mohanty, Chandra (2003): Feminism without Borders: Decolonizing Theory, Practicing Solidarity. Durham, NC.

Moore, Carlos (1982): This Bitch of Life. London: Allison and Busby.

Odim, Cheryl Johnson/Mba, Nina Emma (1997): For Women and Nation: Funmilayo Ransome-Kuti of Nigeria. Urbana-Champagne.

Ogundipe, Molara (1984): African Women, Culture and another Development. In: The Journal of African Marxists 5, 77-92.

Oweyumi, Oyeronke (1997): The Invention of Women: Making an African Sense of Western Gender Discourses. Minneapolis, MN.

Padmore, George (1956): Pan-Afrikanism or Communism. London.

Reddock, Rhoda (2007): Introduction: Diaspora Voices. In: Feminist Africa 7: Diaspora Voices, 1-8.

Reddock, Rhoda (2014): The first Mrs. Garvey and others. Pan-Africaniss and feminism in the early $20^{\text {th }}$ Century British colonial Carribean. In: Feminist Africa Issue 19: PanAfricanism and Feminism, 58-77.

Scott, Bonnie Kime (2007): Gender in Modernism: New Geographies, Complex Intersections. Urbana-Champagne.

Scott, Joan (1986): Gender: A Useful Category of Historical Analysis. In: The American Historical Review 91:5, 1053-1075.

Scott, Joan (1994): Gender: Eine nützliche Kategorie der historischen Analyse. In: Kaiser, Nancy (Hrsg.): Selbst bewusst. Frauen in den USA. Leipzig, 27-75.

Sherwood, Marika et al. (1999): Claudia Jones. A Life in Exile. London: Lawrence and Wishart.

Steady, Filomena (1981): The Black Woman Cross-Culturally. Cambridge, MA: Schenckman.

Sudbury, Julia (Hrsg.) (2005): Introduction. Global lockdown: Race, Gender, and the Prison-Industrial Complex. New York and London.

Wynter, Sylvia (2003): Unsettling the Coloniality of Being / Power/ Truth / Freedom: Towards the Human, After Man, Its Overrepresentation - An Argument. In: The New Centennial Review 3:3, 257-337.

Zetkin, Clara (1929): Erinnerungen an Lenin - Gespräche über die Frauenfrage. Wien / Berlin. 